\title{
Analysis of Longitudinal Diffusion-Weighted Images in Healthy and Pathological Aging: An ADNI Study
}

\author{
Frithjof Kruggel $^{1}$, Fumitaro Masaki ${ }^{1}$, Ana Solodkin ${ }^{2}$ \\ for the Alzheimer's Disease Neuroimaging Initiative \\ 1: Department of Biomedical Engineering \\ 2: Department of Anatomy \& Neurobiology and Neurology \\ University of California, Irvine, USA
}

October 22, 2016

Abbreviated Title: Analysis of Longitudinal DWI

Associate Editor: Mary McKenna

Keywords: voxel-based morphometry, linear mixed-effects modeling, aging, biomarker, longitudinal imaging, Alzheimer's disease.

Corresponding Author:

Frithjof Kruggel

202 Rockwell Engineering Center

University of California, Irvine, CA 92697-2755, USA

Email: fkruggel@uci.edu

Phone: +1-949-824-3729, Fax: +1-949-824-1727

\begin{abstract}
Background \& New Method:

The widely used framework of voxel-based morphometry for analyzing neuroimages is extended here to model longitudinal imaging data by exchanging the linear model with a linear mixedeffects model. The new approach is employed for analyzing a large longitudinal sample of 756 diffusion-weighted images acquired in 177 subjects of the Alzheimer's Disease Neuroimaging initiative (ADNI).

Results and Comparison with Existing Methods:

While sample- and group-level results from both approaches are equivalent, the mixed-effect model yields information at the single subject level. Interestingly, the neurobiological relevance of the relevant parameter at the individual level describes specific differences associated with aging. In addition, our approach highlights white matter areas that reliably discriminate between
\end{abstract}


patients with Alzheimer's disease and healthy controls with a predictive power of 0.99 and include the hippocampal alveus, the para-hippocampal white matter, the white matter of the posterior cingulate, and optic tracts. In this context, notably the classifier includes a subpopulation of patients with minimal cognitive impairment into the pathological domain.

\section{Conclusion:}

Our classifier offers promising features for an accessible biomarker that predicts the risk of conversion to Alzheimer's disease.

Data used in preparation of this article were obtained from the Alzheimer's Disease Neuroimaging Initiative (ADNI) database (adni.loni.usc.edu). As such, the investigators within the ADNI contributed to the design and implementation of ADNI and/or provided data but did not participate in analysis or writing of this report. A complete listing of ADNI investigators can be found at:

http://adni.loni.usc.edu/wp-content/uploads/how to apply/ADNI Acknowledgement List.pdf.

\section{Significance Statement}

This study assesses neuro-degenerative processes in the brain's white matter as revealed by diffusion-weighted imaging, in order to discriminate healthy from pathological aging in a large sample of elderly subjects. The analysis of time-series examinations in a linear mixed effects model allowed the discrimination of population-based aging processes from individual determinants. We demonstrate that a simple classifier based on white matter imaging data is able to predict the conversion to Alzheimer's disease with a high predictive power. 


\section{Introduction}

A vast amount of imaging methods (Braskie et al., 2013) and image-derived metrics (Weiner et al., 2012) have been proposed for the non-invasive, early detection of Alzheimer's diseases (AD) (McKhann et al., 2011). Because AD was primarily assessed via grey matter (GM) degeneration, an initial focus was given on the quantification of GM regions and their metabolic activity. While it is possible to describe the disease process quantitatively in large population samples, detecting correlates of the relative minute brain degeneration in early stages of AD is difficult to achieve at the individual level, due to the limited test-retest precision of current imaging devices and analysis processes. In search of accessible biomarkers for AD that precede a macroscopically measurable brain atrophy, amyloid PET imaging using a $\left[{ }^{11} \mathrm{C}\right] \mathrm{PIB}$ marker (Morris et al., 2009), levels of specific proteins in the cerebro-spinal fluid (CSF) (Villain et al., 2010), and more recently, levels of specific phospholipids in plasma (Mapstone et al., 2014) have so far the best predictive power. Different analytic strategies based on machine learning procedures were developed that use combinations of cortical markers and protein measures for an automatic discrimination of AD patients and controls (e.g., Gao et al., 2014; Liu et al., 2013; Suk and Shen, 2014; Yu et al., 2016; Zu et al., 2016), with correct classification rates up to 96.5\%.

Diffusion-weighted MR imaging (DWI) examines micro-structural properties of white matter (WM). It is well established that the structural integrity of axons and their structural matrix is affected by this degenerative process long before neuronal loss (Hyman et al., 1986; Sadun and Brassi, 1990; Scheltens et al., 1995; Solodkin et al., 2013). There is a rich literature on DWI in healthy and pathological aging (for reviews see Chua et al., 2008; Madden et al., 2012; Sullivan and Pfefferbaum, 2006; Sullivan and Pfefferbaum, 2007) where cognitively normal elderly subjects with positive amyloid beta protein levels were found to have abnormal diffusion metrics (Molineuvo et al., 2014). Converging evidence is documented that metrics based on DWI have high sensitivity for detecting AD-related WM changes (Amlien et al., 2014; Bennett et al., 2010; Douaud et al., 2011; Huang et al., 2012; Kantarci et al., 2005; Keihaninejad et al., 2013; Salat et al., 2010; Teipel et al., 2010; Teipel et al., 2014, Wang et al., 2016; Zhang et al., 2013). In this work, we investigate whether DWI-based metrics are capable biomarkers that provide sufficient power to predict conversion to AD (Douaud et al., 2013; Solodkin et al., 2013) and allow an individual risk assessment. We employ data from a longitudinal study, in order to distinguish between the dynamics of aging- and disease-related influences on WM properties. More specifically, we analyze structural and diffusion-weighted imaging data in a sample of 177 elderly subjects with 2-6 longitudinal examinations collected in the ADNI Go and ADNI 2 studies (ADNI Manual, 2016) over a period between 3 months to 4 years.

Most current studies in time-series analysis of DWI data have employed "tract-based statistics" to extract image metrics in region-of-interests (ROIs) for further statistical analysis (e.g., Nir et al., 2015, Yhan et al., 2015). While such a two-level strategy offers the advantage of abstracting from large data sets in an early processing stage, there is a risk of missing valuable detail due to the focus on a subset of the cerebral WM. Instead, we use voxel-based morphometry (VBM), a widely used framework to assess neuroimaging data in relation to demographic and

clinical features of population samples (Ashburner and Friston, 2000). We replace the linear model (LM) in VBM by a linear mixed-effects model (LMEM), so that single subject-related effects assessed in repeated measurements can explicitly be modeled. In this single-level strategy, we retain the full, voxel-based information in the statistical analysis. Due to the large sample included, we achieve a high statistical power that allows drawing conclusions not only for the WM compartment (as in tract-based statistics), but for GM and composite GM/WM regions as well. Linear mixed-effects models have been a method of choice for the exploratory analysis 
of data acquired in longitudinal studies (Galecki and Burzykowsli, 2013; Laird et al., 1987; Lindstrom and Bates, 1988). We (Kruggel et al., 2010) and others (e.g., Beckmann et al., 2003; Bernal-Rusiel et al., 2013; Driscoll et al., 2009; Resnick et al., 2003; Schumann et al., 2010) have used mixed-effects modeling to assess longitudinal imaging data, based on quantitative features extracted from images rather than directly on voxel-wise data.

In the following section, we formally introduce the image-based measures, the linear mixed-effects models, and describe the study sample and image processing steps. Second, we discuss (a) age- and disease-related influences on WM integrity, (b) demonstrate the advantage of using subject-level information provided by LMEM, and (c) show that static and timedependent measures can be combined to assess the individual risk of converting to Alzheimer's disease $(\mathrm{AD})$. The final section is devoted to an in-depth discussion of the advantages of LMEM, the neuro-biological results obtained, and the potential of an image-based biomarker for the early detection of Alzheimer's disease.

\section{Materials and Methods}

Briefly, we characterize voxel-wise diffusive properties by computing diffusion tensors and their scalar parameters fractional anisotropy (FA), medial and radial diffusivity (MD, RD), in addition to the $\mathrm{T}_{2}$ intensity (T2) as obtained from the gradient-free image. Then, we use the context of voxel-based morphometry to evaluate the dependency of imaging data on clinical and demographical data, but replace the linear model with a linear-mixed effects model to explicitly model subject-wise longitudinal data. The mixed effects model can be understood as an extension of linear regression, where observations are grouped into units, and sample-wide (fixed) effects are separated from unit-wise (random) effects. In this context, a unit corresponds to the longitudinal data acquired in a single subject. Thus, LMEM allows differentiating between sample-level and subject-level effects

\subsection{Image-Based Measures}

Diffusion-weighted MR imaging assesses the direction-dependent diffusion of protons. More specifically, the mobility of extra- and intracellular water molecules hindered by membranes, such as axonal sheaths is examined. The signal $\mathrm{S}(\mathbf{g})$ weighted in gradient direction $\mathbf{g}$ is related to the un-weighted signal $\mathrm{S}_{0}$ by the Stejskal-Tanner equation:

$$
\frac{S(\mathbf{g})}{S_{0}}=\exp -b \mathbf{g}^{T} \mathbf{D g}, \quad \mathbf{D}=\left(\begin{array}{lll}
d_{x x} & d_{x y} & d_{x z} \\
d_{x y} & d_{y y} & d_{y z} \\
d_{x z} & d_{y z} & d_{z z}
\end{array}\right)
$$

where the symmetric, positive semi-definite matrix D captures the voxel-wise anisotropy of water diffusion, and other measurement-related parameters are collected in b. Most commonly, the matrix $\mathbf{D}$ is decomposed into its three eigenvectors $\left(\mathbf{e}_{1}, \mathbf{e}_{2}, \mathbf{e}_{3}\right)$, corresponding to the main directions of water motion, and its three eigenvalues $\left(\lambda_{1}, \lambda_{2}, \lambda_{3}\right)$, representing the amount of diffusion in each direction. Voxel-wise diffusive properties are often expressed by scalar measures fractional anisotropy (FA), mean diffusivity (MD) and radial diffusivity (RD): 


$$
\begin{aligned}
\mathrm{FA} & =\sqrt{\frac{3\left(\left(\lambda_{1}-\overline{\lambda_{1}}\right)^{2}+\left(\lambda_{2}-\overline{\lambda_{2}}\right)^{2}+\left(\lambda_{3}-\overline{\lambda_{3}}\right)^{2}\right)}{2\left(\lambda_{1}^{2}+\lambda_{2}^{2}+\lambda_{3}^{2}\right)},} \\
\mathrm{MD} & =\frac{1}{3}\left(\lambda_{1}+\lambda_{2}+\lambda_{3}\right), \\
\mathrm{RD} & =\frac{1}{2}\left(\lambda_{2}+\lambda_{3}\right)
\end{aligned}
$$

Higher FA values indicate a larger anisotropy, typically found in intact "unidirectional" fiber bundles. Higher MD values correspond to a larger overall mobility of water, often related to a higher fraction of extra-cellular water. RD characterizes the amount of diffusion radial to a fiber bundle, with higher values indicating a lower fiber integrity ("leakiness"). Note that voxels with more than one fiber compartment are expected to have a lower FA (i.e., multiple fiber directions) and a higher MD (i.e., a larger fraction of extracellular space). As an additional, independent indicator of WM degeneration, we included the signal of the T2-weighted images in our analysis, which is roughly proportional to the fraction of extracellular water.

\subsection{The Linear Mixed-Effects Model}

The general form of the linear mixed effects model is written as (Laird et al., 1987):

$$
\mathbf{y}=\mathbf{X} \beta+\mathrm{U} \gamma+\boldsymbol{\epsilon}, \quad\left(\begin{array}{l}
\gamma \\
\epsilon
\end{array}\right) \sim \mathcal{N}_{m q+n}\left(\left(\begin{array}{l}
0 \\
0
\end{array}\right),\left(\begin{array}{cc}
\mathrm{G}(\boldsymbol{\theta}) & \mathbf{0}_{m q \times n} \\
0_{n \times m q} & \mathbf{R}(\boldsymbol{\theta})
\end{array}\right)\right)
$$

with covariance vector $\boldsymbol{\theta}$. A vector of $\mathrm{n}$ measurements $\mathbf{y}$ is modeled by a $n \times p$ dense matrix $\mathbf{X}$ of fixed effects and their $p$ regression coefficients $\boldsymbol{\beta}$, an $n \times(m q)$ block-diagonal sparse matrix $\mathbf{U}$ of random effects and their $(m q)$ regression coefficients $\gamma$ and an additive error term $\boldsymbol{\varepsilon}$. In our application, $m$ corresponds to the number of units (e.g., subjects) included in the study, each with $n_{i}$ time series measurement, i.e., $n=\sum_{i=1}^{m} n_{i}$. Dropping the random effects term $\mathbf{U} \gamma$ leads to a linear model. Using $\mathbf{V}(\boldsymbol{\theta})=\mathbf{U} \mathbf{G}(\boldsymbol{\theta}) \mathbf{U}^{\mathrm{T}}+\mathbf{R}(\boldsymbol{\theta})$, the fixed effects $\boldsymbol{\beta}$ and the random effects $\boldsymbol{\gamma}$ are estimated by:

$$
\hat{\beta}=\left(\mathbf{X}^{T} \mathbf{V}(\hat{\boldsymbol{\theta}})^{-1} \mathbf{X}\right)^{-1} \mathbf{X}^{T} \mathbf{V}(\hat{\boldsymbol{\theta}})^{-1} \mathbf{y} \text { and } \hat{\gamma}=\mathbf{G} \hat{\mathbf{U}}^{T} \mathbf{V}(\hat{\boldsymbol{\theta}})^{-1}(\mathbf{y}-\mathbf{X} \hat{\boldsymbol{\beta}}) .
$$

The covariance vector is typically estimated using Restricted Maximum Likelihood (REML) by:

$$
\hat{\boldsymbol{\theta}}=\arg \max \left(l_{p}(\boldsymbol{\theta})-\frac{1}{2} \ln \left|\mathbf{X}^{T} \mathbf{V}(\boldsymbol{\theta})^{-1} \mathbf{X}\right|\right) .
$$

For the estimation of the model parameters $\boldsymbol{\beta}, \boldsymbol{\gamma}_{\mathrm{i}}$ and inference on $\boldsymbol{\beta}$, refer to (Halekoh and Hojsgaard, 2014; Kenward and Roger, 1997; Laird et al., 1987). In order to make the problem identifiable, we make the simplifying assumptions $\gamma \sim \mathrm{N}_{\mathrm{q}}(0, \mathbf{D})$ and $\varepsilon \sim \mathrm{N}_{\mathrm{n}}\left(0, \sigma^{2} \mathbf{I}\right)$ so that the covariance vector $\boldsymbol{\theta}$ has $s=q(q+1) / 2+1$ parameters. Note that the conditions $n \geq q+m q+s$ and $n_{i} \geq$ $q$ pose an upper limit on the number of fixed and random regressors $p, q$.

In our application, $\mathbf{y}$ corresponds to a vector of $n$ voxel-wise measures collected at the brain regions of interest in the study sample. The matrix $\mathbf{X}$ contains $p$ regressors with grouprelated information (e.g., disease status, sex) and subject-level information (e.g., age). Each submatrix $\mathbf{U}_{\mathrm{i}}$ includes information about longitudinal data of subject $i$ (e.g., the time interval between

examinations). As a result, we obtain voxel-wise estimates of the regression coefficients $\widehat{\boldsymbol{\beta}}$ and 
their significance levels $\widehat{\boldsymbol{p}}$ are compiled in spatial parameter maps. These parameters correspond to sample-level effects (e.g., WM integrity as a function of aging), and group-level effects (e.g., differences in WM integrity between patients and healthy controls). In addition, we obtain voxeland subject-wise estimates of regression coefficients $\gamma_{i}$ that provide local and individual information about WM integrity, relative to the sample average.

\subsection{Subjects}

This work includes all subjects in the ADNI Go and ADNI 2 studies for which two or more diffusion-weighted MRI scans were available. Besides the diffusion-weighted data set, we also included $\mathrm{T}_{1}$-weighted $\mathrm{MR}$ images acquired in the same session. After finding and removing incomplete and low-quality data sets, 177 subjects remained (69 females, 108 males, aged between 48.7 and 90.9 years, mean 74.6 years). Written informed consent was obtained for participation in these studies, approved by the institutional review board at each of the 15 participating centers. A total of 756 examinations were performed between June 2010 and January 2016, corresponding to 2-6 time series examinations per subject. Most typically, intervals between exams were 3 months, 6 months, followed by yearly intervals, although intervals varied considerably. The total observation per included subject ranged between 20 and 1280 days. In addition to the MR images, demographic data (e.g., age, weight, gender) and cognitive scores were collected. At time of enrollment, subjects were classified to 5 clinical groups CN: cognitively normal $(n=43)$; EMCI: "early" mild cognitive impairment $(n=58)$; LMCI: "late" MCI $(n=33)$; SMC: severe memory complaint $(n=7)$; AD: Alzheimer's disease $(n=36)$. These categories were defined as follow (see ADNI Manual, 2016, p.27): CN subjects were (a) free of memory complaints, (b) showed normal memory function in the Logical Memory II subscale from the Wechsler Memory Scale, (c) had an MMSE score at or above 24, and (d) had a clinical dementia rating (CDR) of 0 . The classification of AD was based on: (a) memory complaints, (b) abnormal memory function in the Wechsler test, (c) a MMSE score at or below 24, and (d) a dementia rating (CDR) of 0.5 or greater. Subjects classified as any of the MCI groups ranged between these extremes, where EMCI subjects had a better overall result in the memory test than LMCI subjects. Age and gender did not significantly differ between groups $(p>0.05)$. Results of clinical examinations and diagnostic labels were sampled until September 2016. Of the $98 \mathrm{MCI}$ patients included here, 30 (30.6\%) had their diagnosis revised: 26 (26.5\%) converted to $\mathrm{AD}$, and 4 reverted to $\mathrm{CN}$.

Data used in the preparation of this article were obtained from the Alzheimer's disease Neuroimaging Initiative (ADNI) database (adni.loni.usc.edu). The ADNI was launched in 2003 as a public-private partnership, led by Principal Investigator Michael W. Weiner, MD. The primary goal of ADNI has been to test whether serial magnetic resonance imaging (MRI), positron emission tomography (PET), other biological markers, and clinical and neuropsychological assessment can be combined to measure the progression of mild cognitive impairment (MCI) and early Alzheimer's disease (AD).

\subsection{Imaging Protocol}

All 15 participating sites were equipped with General Electric Healthcare scanners, models Discovery or Signa. $\mathrm{T}_{1}$-weighted images were acquired using a 3D IR-FSPGR protocol (TE $3.036 \mathrm{~s}$, TR $7.34 \mathrm{~s}$, TI $400 \mathrm{~ms}$ ) using a $256 \times 256$ matrix, 196 sagittal slices, $1.0156 \mathrm{~mm}$ inplane resolution, $1.2 \mathrm{~mm}$ slice thickness. Diffusion-weighted images were acquired using an EPI SE protocol (TE $90 \mathrm{~ms}$, TR $9.05 \mathrm{~s}$ ) using a $256 \times 256$ matrix, 59 axial slices, $1.37 \mathrm{~mm}$ in-plane 
resolution, $2.7 \mathrm{~mm}$ slice thickness, 41 diffusion-weighted volumes at a b-value of $1000 \mathrm{~s} / \mathrm{mm}^{2}, 5$ isotropic volumes. For further details, see (ADNI MRI Scanner Protocols, 2016).

\subsection{Image Analysis}

The first sequence of image processing steps aimed at generating a study-specific anatomical template from $\mathrm{T}_{1}$-weighted imaging data: (A1) $\mathrm{T}_{1}$-weighted imaging data in DICOM format were converted into BRIAN format. The first data set of each time series was aligned with the stereotactic coordinate system (Kruggel and von Cramon, 1999a) and interpolated to an isotropic voxel size of $1 \mathrm{~mm}$ using a fourth-order b-spline method. Subsequently, the rest of the data sets were aligned with the first one by linear registration. Quality control parameters (e.g., signal-to-noise, contrast-to-noise, image acuity) were computed for all aligned images. Data sets with quality measures in the lowest $10 \%$ range were visually assessed, and replaced by alternate scans. (A2) All data sets were registered with the ICBM 2009c template (Fonov et al., 2009) using non-linear registration (Vercauteren et al., 2009). All registered head images were averaged, correcting for the mean intensity. The brain was extracted from the average to yield the initial brain template. (A3) A mask of the intracranial volume was generated from each data set by a registration approach and used to remove the outer hulls of the brain (Hentschel and Kruggel, 2004). A block-wise local intensity-based segmentation was used to correct for global intensity inhomogeneities. (A4) All intensity-corrected brain images were non-linearly registered with the initial template, and averaged to yield the study-specific brain template. Importantly, the non-linear deformation field was saved for the trans-formation of the diffusion-weighted data.

Diffusion-weighted imaging data were processed by the following pipeline: (D1) Scan data in DICOM format were converted into BRIAN format. Image volumes corresponding to all gradient directions were corrected for eddy-current artifacts and registered with the gradient-free $\left(\mathrm{T}_{2}\right.$ )-weighted image volume using affine registration and normalized mutual information (NMI) as image similarity metric (Maes et al., 1997). (D2) Diffusion tensors were computed by a nonlinear procedure including anisotropic noise filtering (Fillard et al., 2007) and converted into scalar images of fractional anisotropy (FA), radial and mean diffusivity (RD, MD) (Basser and Pierpaoli, 1996) (D3) The gradient-free images were linearly registered with the aligned $\mathrm{T}_{1^{-}}$ weighted image (step A3) that was acquired at the same examination date as the diffusionweighted scan using NMI as similarity metric. The obtained transformation parameters were applied to the scalar images. (D4) The subject- and time-point specific non-linear deformation fields (step A4) were used to transform all FA, RD, MD, T2 images into stereotactic space, smoothed by a Gaussian filter $(\sigma=2$, FWHM of $4.7 \mathrm{~mm})$, and masked against the study-specific brain template (step A4).

All four image series (T2, FA, RD, MD) were analyzed by LMEM models, using a $n \times p$ matrix of study-wide and a $n \times q$ matrix of subject-specific covariates. Resulting voxel-wise estimates for the $\mathrm{p}$ regression parameters $\boldsymbol{\beta}_{\mathrm{i}}$, and their corresponding significance levels were compiled as $\mathrm{p}$ volumetric parameter images. In order to correct for multiple comparisons, we either assessed supra-threshold clusters in $z$-score images (Friston et al., 1993), or corrected significance levels based on a permutation test (Benjamini and Hochberg, 1995). Note that the computation was restricted to a brain tissue (GM and WM) mask segmented from our studyspecific template (A4).

Image processing was performed on a 30-node computer cluster equipped with AMD 64 two-core CPUs, 4 GB of memory, running under the Debian 8/Linux 3.16 operating system, using the BRIAN image analysis environment (Kruggel, 2016). We refer to "VBM framework" here as a variant of the techniques described in (Ashburner and Friston, 2000), implemented in 
efficient compiled code and more recent image processing techniques. For performing logistic regression, principal component analysis (PCA), and receiver-operating characteristics (ROC), we used the R system (Hornik, 2014). Neuroanatomical labels were addressed to GM structures and WM fiber bundles via coordinates in standardized space by consulting a neuroanatomical atlas (Cho, 2010).

\section{$3 \quad$ Results}

Mixed effects models allow differentiating between population- and subject-level effects which is necessary for an individual risk assessment in neurodegeneration. First, we describe the population- and disease-related influences on WM degeneration. Next, we derive a subject-level measure that captures aspects of the individual aging process. Finally, we demonstrate how all imaging information can be integrated into a simple classifier that allows distinguishing between healthy controls and subjects with AD at a high level of accuracy.

\subsection{Determinants of Healthy and Pathological Aging in WM}

We considered a LMEM with the following fixed covariates: age at the time of each examination (AGE), gender (GEN), clinical group (GRP), and brain volume (BRV):

$$
y=\beta_{0}+\beta_{1} \mathrm{AGE}+\beta_{2} \mathrm{GEN}+\beta_{3} \mathrm{GRP}+\beta_{4} \mathrm{BRV}+\gamma_{0}+\gamma_{1} \mathrm{AGE} .
$$

Because individual brain data were mapped into a common space, brain volume is included here as a nuisance regressor.

Age-related effects: To ease the understanding of the spatial continuity of results for age-related fixed effects, we did not threshold the $z$-score maps. Fig. 1 shows un-thresholded z-score maps (positive range from $0=$ red to $8=$ white, negative range from $0=$ green to $-8=$ magenta), overlaid onto the study-specific anatomical template. Tab. 1 collects the location, Talairach coordinates, age-related coefficients $\beta$ for measures FA, RD, MD, and T2, their standard deviation, and significance (expressed as z-score) for selected locations.

\section{$* * * * * \quad$ INSERT FIGURE 1 ABOUT HERE $* * * * *$ \\ $* * * * * \quad$ INSERT TABLE 1 ABOUT HERE $* * * * *$}

We note:

- There was a generalized age-related decrease of FA in the WM, most nuanced in the ventral portions of both frontal lobes, and rostral-to-medial portions of the corpus callosum. These areas showed the strongest age-related increase of RD, MD, and T2 (pattern 1).

- Conversely, a strong age-related increase of FA, with a moderate decrease in RD, MD, and T2 was found in the GM, most prominently, in insula, basal ganglia, and the cingulate gyrus (pattern 2).

Several studies have assessed the effects of aging onto WM structures of the human brain (for reviews see Madden et al., 2012; Sullivan and Pfefferbaum, 2006). A general trend across many investigations points to a decrease in FA and an increase in RD and MD with increasing age in adults (pattern 1), corresponding to a decline in WM composition and integrity, with demyelinization as a leading factor (Madden et al., 2012). This pattern is not homogeneous throughout the brain. Besides pattern 1, a strong increase in FA, with a moderate decrease in RD and MD (pattern 2) was found in some GM structures (insula, putamen, pallidum, and the 
cingulate gyrus). The mechanisms suggested behind this pattern include gliosis, tissue compaction, increased iron deposits (Pfefferbaum et al., 2010; Wang et al., 2010). We prefer the interpretation of a degenerative cytotoxic edema in GM (Lin et al., 2016), because this explanation matches well with the overall occurrence of the affected regions.

Gender-related effects: We show thresholded $z$-score maps in Fig. 2 (positive range from $3=$ red to $8=$ white, negative range from $-3=$ green to $-8=$ magenta), overlaid onto the study-specific anatomical template. Only regions are shown that were confirmed as significantly different after correction for multiple comparison by controlling the family-wise error rate (Friston et al., 1993), given a cluster selection threshold of $\mathrm{p}<0.01$. Tab. 2 collects the location, Talairach coordinates, supra-threshold cluster size (in $\mathrm{mm}^{3}$ ), peak and mean z-scores for significantly different regions.

***** INSERT FIGURE 2 ABOUT HERE *****

$* * * * * \quad$ INSERT TABLE 2 ABOUT HERE $* * * *$

We note:

- Females had significantly higher FA, lower MD and RD in the splenium of the corpus callosum, the inferior fronto-occipital tract on both sides, the superior cerebellar peduncle, nigro-striatal pathways, and in WM of the rostral cerebellum. This pattern is, most likely, due to the larger density and smaller diameter of fiber connections in females.

- Females also had lower FA, higher RD and MD in cortico-fugal fibers and in WM of the caudal cerebellum on both sides. In addition, females had a significantly lower FA and higher T2 in the ventral pallidum, the nucleus accumbens, and it's mid- and forebrain connections on both sides. These findings point towards a larger fraction of extra-cellular water in these regions, although the neuroanatomical basis is unclear.

While sexual dimorphism in grey matter is well described, relatively little focus has been given to white matter (Hsu et al., 2008; Kovalev et al., 2003), until recently where substantial differences have been described (Ingalhalikar et al., 2014; Kanaan et al., 2012; Kanaan et al., 2014). Yet, converging results on specific brain regions and the sign of the difference are still missing. Qualitatively, we find a match between our maps and those published by Inano et al. (2011), with the sign reversed, that is, we found a higher FA in females in the splenium of the corpus callosum, in agreement with some (Ingalhalikar et al., 2014; Kanaan et al., 2012; Kanaan et al., 2014) but not Inano et al. (2011). Likewise, we found a higher FA in females in the superior cerebellar peduncle and WM in the anterior cerebellum, which was not significant in previous publications (Kanaan et al., 2012; Kanaan et al., 2014). In addition, females had a significantly lower FA and higher T2 in the globus pallidus, the nucleus accumbens, and it's midand forebrain WM connections on both sides. This may have been un-noticed before because most other studies used tract-based statistics for analysis, and these regions lie outside the tract atlas. In addition, our population consists of elderly subjects only, while others are based on a broad age range of adults. A thorough assessment of sex-related differences is a subject for future work.

Disease-related effects of AD vs. healthy controls: As for the age-related effects, we did not threshold the $z$-score maps, to ease the understanding of the spatial continuity of results. Fig. 3 shows un-thresholded $\mathrm{z}$-score maps (positive range from $0=$ red to $8=$ white, negative range from $0=$ green to $-8=$ magenta), overlaid onto the study-specific anatomical template. Tab. 3 collects the location, Talairach coordinates, age-related coefficients $\beta$ for measures FA, RD, MD, and T2, their standard deviation, and significance (expressed as Z-score) for selected locations. 
***** INSERT FIGURE 3 ABOUT HERE *****

$* * * * *$ INSERT TABLE 3 ABOUT HERE *****

We note:

- The main effect was a strong, bilateral disease-related increase of RD and MD in the corpus callosum and hippocampus with a barely significant decrease in FA and increase in T2 (pattern 3).

- A less prominent, disease-related increase of FA and T2, without significant changes in RD and MD was seen in cortico-fugal fibers associated with the pyramidal tract and corona radiata at the level of the supra-ventricular WM, predominant on the left side (pattern 4).

The disease-related pattern 3 parallels previous work (see Chua et al. (2008) for a review). These changes are best explained by a disease-related increase in extra-cellular water, likely due to vasogenic edema (Lin et al., 2016). Regions with the strongest disease-related differences are found bilaterally in the ventro-medial temporal lobe, and in posterior sections of the corpus callosum. Recently, Douaud et al. (2011) reported regions of increased FA in the centrum semiovale which is reproduced in similar extent and location here as pattern 4 . We find that this pattern is quite extensive, albeit at a lower significance than pattern 3. As indicated by Bennett et al. (2010) and Douaud et al. (2011), we hypothesize that in those regions with crossing fiber compartments, one degenerates slower than other(s). Our results are in agreement with the fact that the sensorimotor cortical GM and descending cortico-spinal tract remain relatively intact in AD (Scheltens et al., 1995).

\subsection{Measures of Individual Aging in WM}

This was possible as in our mixed model the model (Section 3.1), age is included as both a population-level effect $\beta_{1}$ and as a subject-level effect $\gamma_{1}$. Thus, individual differences in an image metric are explained as $\Delta y=\gamma_{0}+\gamma_{1}$ AGE, obtained from the time-series measurements in the particular subject. Let us consider the hypothesis that this difference is due to an unobserved individual difference in the aging process, termed as $\triangle \mathrm{AGE}$ :

$$
\Delta \mathrm{AGE}=\frac{1}{\beta_{1}}\left(\beta_{4}(\mathrm{BRV}-\overline{\mathrm{BRV}})+\gamma_{0}+\gamma_{1} \overline{\mathrm{AGE}}\right),
$$

where bars denote subject-wise means. Higher (positive) values indicate that tissue in this voxel aged more than the sample average, while lower (negative) values indicate that tissue in this voxel is less affected by aging. We restricted the computation of $\triangle \mathrm{AGE}$ to those voxels in which age-related changes are significant $\left(p<0.05\right.$, as determined for $\beta_{1}$ ). Empirical distributions of $\triangle$ AGE pass tests for uni-modality (Johnson and Rogers, 1951), indicating that there is a single process generating the data. However, distributions have "heavy tails" on both sides, compared to a Gaussian distribution.

Next, we averaged results across all voxels of a subject. Thus, $\triangle \mathrm{AGE}$ is now used as a subject-wise measure. The resulting measures, as computed independently from T2 intensities and DTI parameters FA, RD, MD show a high correlation, and a slope close to 1 (for T2, around 0.5), (Tab. 1). Hence, $\triangle \mathrm{AGE}$ captures essentially the same biophysical properties when computed from FA, RD, and MD data, and similar properties when computed from T2-weighted data. 
We then performed a linear regression to compute the relationship between $\triangle \mathrm{AGE}$ and the demographic and clinical data of each subject. Variables AGE, GEN, and brain volume did not significantly influence $\triangle \mathrm{AGE}$, confirming that our procedures successfully parceled out these covariates. However, the clinical groups $\mathrm{AD}(+3.82, p=0.0005)$, EMCI $(+3.15, p=0.0014)$ and LMCI $(+2.99, p=0.0081)$ differed significantly in their $\triangle$ AGE from healthy controls and subjects with severe memory complaints, although this model explains only $8.4 \%$ of the variance. Likewise, the MMSE scores were correlated with $\triangle \mathrm{AGE}(-0.345, p=0.00355)$, suggesting that the measure $\triangle \mathrm{AGE}$ separates aspects of individual aging from the general (population-level) aging process.

Finally, we studied how the mixed-effects modeling helps estimate a "prototypical" aging function from empirical data. We selected the first (FA, RD, MD, T2) data set from each subject, and averaged measures across all voxels in which a significant age-dependency was found $(p<$ 0.05). A plot of FA vs. subject age (Fig. 4, top left, red circles) shows a clear age-dependent trend with a considerable inter-subject variability. Adjusting a subject's real age by the estimated individual difference of the aging process, $\triangle \mathrm{AGE}$, reduces the inter-individual variance (shown as black dots), i.e., measurements represented as red circles are shifted horizontally by the correction to the black dots. For the age range in this study, the dependence of a measured parameter can be approximated by a linear relationship (Fig. 4; FA: $-0.025 /$ year, $\mathrm{R}^{2}=0.92$; RD: $+0.0094 /$ year, $\mathrm{R}^{2}=0.84$; $\mathrm{RD}:+0.0094 /$ year, $\mathrm{R}^{2}=0.85 ; \mathrm{T} 2:+30.1 /$ year, $\mathrm{R}^{2}=0.72$, all $p \sim 0$ ).

\section{***** INSERT FIGURE 4 ABOUT HERE *****}

Thus, the linear mixed effects model is able to separate the individual from the population-level variance. Adjusting population data by individual factors leads to better population-based models. These findings also confirm that the initial assumption of a linear dependency of these measures on age is warranted.

\subsection{Individual Image-Based Diagnosis}

To illustrate the diagnostic power of our methods, we have built an image-based classifier to assess the individual risk of converting to $\mathrm{AD}$. We assume that brains of $\mathrm{CN}$ subjects follow a "healthy aging" process, and brains of AD patients a "pathological aging", with region- and agedependent properties as discussed above, and consider their diagnostic label as true and independent of time. We use this diagnostic information as "ground truth" to build a classifier that distinguishes healthy from pathological aging. For the brains of the MCI patients in our sample, we assume that their aging process falls into the domain spanned by the two extremes above. To assess the individual risk of converting to $\mathrm{AD}$, we rate their aging process using our image-based classifier.

To describe the individual properties of aging tissue in a voxel, we used the intercept at the mean sample age $\left(\beta_{0}+\gamma_{0}+\left(\beta_{1}+\gamma_{1}\right) \overline{\mathrm{AGE}}\right)$ - to represent the individual state of a tissue property at a normalized age, and the subject-specific age-related slope $\left(\beta_{1}+\gamma_{1}\right)$ - to represent the individual change of a tissue property per time unit. Both values were computed for each measure (FA, RD, MD, T2), yielding a vector of eight values per voxel. We sampled voxel-wise properties from a region-of-interest in the anterior mesial temporal lobe comprised on WM and GM, with a size of 19500 voxels. WM fibers in this region are affected first by AD, as found from histopathology (Scheltens et al., 1995) and our prior knowledge (Solodkin et al., 2013). 
These eight parameters were averaged within a subject, and z-transformed per parameter, yielding 8 normalized measures per subject. Observations from healthy controls and AD patients were used to build a classifier using logistic regression, using the diagnostic label as independent, and the subject-wise measures as dependent variables. Predictions from the model have a high discriminative power of 0.991 , as assessed by the "area under the curve" (AUC) of a receiveroperating characteristics (ROC) curve. A repeated random sub-sampling cross-validation with a $20 \%$ test set yielded a correct prediction rate of 95.4\% (95\% CI: 0.90-1.0). Applying this classifier to all groups, the following fractions were classified as pathological: AD: 97.2\% (35/36); EMCI: $15.5 \%$ (9/58); LMCI: $42.8 \%$ (14/33); CN: 4.6\% (1/22); SMC: 14.3\% (1/7) (Fig. $5)$. Within the observation time frame, 30/91 MCI patients had their diagnosis revised. Of the 23 MCI patients addressed to the pathological domain, 21 (91.3\%) converted to AD. Of the $68 \mathrm{MCI}$ patients addressed to the healthy domain, four $(5.9 \%)$ reverted to $\mathrm{CN}$, and five $(7.4 \%)$ converted to AD. Classifiers for the distinction of LMCI vs. AD yielded a discriminative power of 0.943 , for EMCI vs. AD: 0.833.

\section{***** INSERT FIGURE 5 ABOUT HERE *****}

The voxels that discriminate best between healthy controls and AD patients were found in overprojection with white matter fiber structures of the hippocampus and parahippocampal regions, e.g., the alveus and the parahippocampal white matter containing the perforant pathway (Fig. 6). Somewhat surprisingly, the optic tract on both sides was also found as discriminatory.

***** INSERT FIGURE 6 ABOUT HERE *****

A more parsimonious classifier can be constructed by replacing the z-transformation above with a Principal Component Analysis (PCA), yielding a space spanned by linear combinations of the original eight parameters, with dimensions sorted by their relative importance (i.e., their contribution to the overall variance). We mapped our subject-wise observations into this space, and perform the logistic regression. Here, we can select the dimension(s) that have the highest discriminatory power. Using just one dimension (or regressor), we still found a high predictive power of 0.952 (AUC).

Any other ROI can be devised to build a classifier. A more data-driven approach may start from the disease-related z-maps obtained in Section 3.1, and select those voxels that most highly discriminate between $\mathrm{AD}$ and NC. We defined a region in the middle and posterior corpus callosum, and obtained a classifier with a considerably lower discriminatory power (0.894). A potential explanation is that this region is based on time-independent information (fixed-effects) only, and that dynamic information (from random effects) contributes much to the decision process. More research on the optimization of the region selection is underway. 


\section{Discussion}

In this study, we extended the well-established framework of voxel-based morphometry to the analysis of data from longitudinal imaging studies. We used a large sample of longitudinal DW images acquired in elderly subjects and demonstrated the high statistical power to pinpoint age-related degeneration patterns in WM, gender-related differences, and patterns related to Alzheimer's disease.

In addition to the assessment of population-level effects, we demonstrate that individualized, subject-level results obtained in a mixed-effects linear model offer additional, neuro-biologically relevant information. In this context we showed how to differentiate general (population-level) aging processes from modulating individual factors.

Pathological signs of WM degeneration obtained in this study are highly discriminative, since a classifier for potential conversion to AD achieved a high predictive power of 0.991 , which is among the highest values currently published (Gao et al., 2014; Kohannim et al., 2010; O'Dwyer et al., 2011; Ravetti and Moscato, 2008, Vlassenko et al., 2016; Zu et al., 2016). That is, this classifier provided the membership of some MCI patients into the pathological domain, which are likely candidates for conversion to AD.

To put these contributions into context, it is important to examine the following:

\subsection{Mixed-Effects Modeling of DWI Time Series Data}

Technological development is allowing us to move towards precise, individualized medicine. Hence, the logical extension from linear to mixed models offers a distinction between population- to subject-level effects. However, this extension has a price: while regression coefficients in a linear model are directly computed (using the pseudo-inverse of $\mathbf{X}$ ), the estimation process in mixed-effects models is inherently iterative, requiring considerably more computational resources. In consequence, most groups so far have used LMEM in relatively small scale analyses on image-derived measures (e.g., Kruggel et al., 2010) or tract-based statistics (Teipel et al., 2007). Recently, an analysis of longitudinal data on neocortical thickness demonstrated the feasibility of this approach (Bernal-Rusiel et al., 2013). That is, an efficient implementation of the estimation algorithm is essential - and in consequence- we developed an optimized code. Because most operations work on small matrices, porting this code to a massparallel graphics processing unit is expected to achieve further gains in speed. However, we found that sample- and group-level results of linear regression were equivalent to those obtained by a mixed-effects model. Thus, a first exploratory approach may use linear models to assess covariates and determine the best composition of $\mathbf{X}$ before moving onto LMEM. Results of a LM can also be used to define regions-of-interest on which are more extensive LMEM is computed.

Note that we considered voxel-wise estimations as spatially independent. Extensions of linear models that take spatial autocorrelations into account have been developed for functional MRI (Katanoda et al., 2002; Kruggel and von Cramon, 1999b). Essentially, a small neighborhood of voxels is considered, and the voxel-wise estimators are "stacked up" to form block-diagonal sparse matrices, connected by a spatial autocorrelation matrix. Because this extension will increase the computation time at least by an order of magnitude, we followed the common practice in voxel-based morphometry and apply either a cluster-level correction (Friston et al., 1993) or a permutation test to control the family-wise error rate (Benjamini and Hochberg, 1995).

Whereas most DWI studies use tract-based statistics to extract image-based metrics early 
in the analysis process, and conduct inferential analyses at a symbolic (e.g., region) level, our approach is image-based, exploratory, and requires few assumptions. Extending the linear model used in voxel-based morphometry to mixed-effects model is conceptually simple, and allows differentiating between population-, group-level and individual properties.

\subsection{WM Degeneration as a Biomarker for AD}

The static and dynamic (time-dependent) image-based measurements can be combined to select regions that are highly discriminative between AD patients and healthy controls. Focusing on the mesial temporal lobe, these include WM structures of the hippocampal formation complex (including the perforant path and alveus), and surprisingly interestingly, the optic tract on both sides. Age- and AD-related changes of the alveus and perforant path are clearly documented by histological (e.g., Arnold et al., 1991; Hyman et al., 1986), and more recently, DWI investigations (Damoiseaux et al., 2009; Fellgiebel and Yakushev, 2011; McEvoy et al., 2009; Rogalski et al., 2009; Salat et al., 2010; Solodkin et al., 2013; Yassa et al., 2010). It was demonstrated that image-based measures are capable of predicting the conversion from MCI to AD (Douaud et al., 2013; Kantarci et al., 2005; Solodkin et al., 2013). Recently, several investigations assessed longitudinal diffusion-weighted data in healthy and pathological aging (Douaud et al., 2011; Douaud et al., 2013; Keihaninejad et al., 2013; Kitamura et al., 2013; Sexton et al., 2014; Teipel et al., 2010). Sexton et al. (2014) performed a tract-based analysis of longitudinal DWI data in subjects with an age range of 20 to 84 years. They report an overall age-related change of diffusion measures corresponding to our pattern 1, predominantly in the frontal and parietal WM that is accelerating beyond the $5^{\text {th }}$ decade of life, with an approximately linear change in FA, RD and MD. Our approach, using a voxel-wise mixed-effects model, offers a higher spatial resolution, does not bias for specific anatomical regions, and allows assessing individual properties. Douaud et al. (2013) studied a group of 56 amnestic MCI patients, of which some converted to AD, others remained stable over a course of three years. They conclude that predicting progression to AD (based on imaging and CSF protein markers) is possible 2-3 years before clinical symptoms occur,

Our results confirm that DWI-based measures show a high sensitivity for detecting WM degeneration in elderly subjects. Introducing a linear-mixed effects model in the context, we demonstrated that the parameter DAGE, derived from repeated measurement, allows measuring differences in the speed of individual aging w.r.t. the sample. Focusing on the WM of the ventromesial temporal lobe, we were able to construct an image-based classifier that predicts the conversion from MCI to AD with a precision of better than $91 \%$, which is comparable to other approaches based on CSF protein biomarkers (Villain et al., 2010), image-based markers (Morris et al., 2009), or a combination of both (Douaud et al., 2013). On an interesting sideline, the optic tract was also found as highly discriminative. While it has been demonstrated that the optic nerve is affected in AD (Hinton et al., 1986; Sadun and Brassi, 1990) we are unaware of any DWI studies that have assessed this structure.

Through this study, we hope to further promote using DWI-based parameters as a biomarker for the early detection of AD. We are aware of a few limitations of this study: (a) we understand that clinical assessments in the ADNI study were not taken at the same time and interval as the image acquistion; (b) the diffusion-weighted imaging protocol may be optimized in terms of spatial resolution, field-of-view, and correction of spatial distortions; (c) replacing the global with an individually defined ROI may enhance the performance of the classifier; (d) while the sensitivity of these measures is repeatedly demonstrated, their specificity (in terms of a differential diagnosis) is unclear. A future study may consider these issues in its design. 
To conclude, exchanging the linear model in voxel-based morphometry by a linear-mixed effects model leads to a "natural" extension that allows a statistically rigorous assessment of longitudinal imaging studies while retaining its conceptual simplicity. Separating subject-level effects leads to better sample-level models, as shown for the prototypical WM aging curves here. Relating individual features with respect to a corresponding clinical group (e.g., MCI patients) allows identifying patients with a high risk for converting to AD.

\section{Acknowledgment}

Data collection and sharing for this project was funded by the Alzheimer's Disease Neuroimaging Initiative (ADNI) (National Institutes of Health Grant U01 AG024904) and DOD ADNI (Department of Defense award number W81XWH-12-2-0012). ADNI is funded by the National Institute on Aging, the National Institute of Biomedical Imaging and Bioengineering, and through generous contributions from the following: AbbVie, Alzheimer's Association; Alzheimer's Drug Discovery Foundation; Araclon Biotech; BioClinica, Inc.; Biogen; BristolMyers Squibb Company; CereSpir, Inc.; Eisai Inc.; Elan Pharmaceuticals, Inc.; Eli Lilly and Company; EuroImmun; F. Hoffmann-La Roche Ltd and its affiliated company Genentech, Inc.; Fujirebio; GE Healthcare; IX-ICO Ltd.; Janssen Alzheimer Immunotherapy Research \& Development, LLC.; John-son \& Johnson Pharmaceutical Research \& Development LLC.; Lumosity; Lundbeck; Merck \& Co., Inc.; MesoScale Diagnostics, LLC.; NeuroRx Research; Neurotrack Technologies; Novartis Pharmaceuticals Corporation; Pfizer Inc.; Piramal Imaging; Servier; Takeda Pharmaceutical Company; and Transition Therapeutics. The Canadian Institutes of Health Research is providing funds to support ADNI clinical sites in Canada. Private sector contributions are facilitated by the Foundation for the National Institutes of Health (www.fnih.org). The grantee organization is the Northern California Institute for Research and Education, and the study is coordinated by the Alzheimer's Disease Cooperative Study at the University of California, San Diego. ADNI data are disseminated by the Laboratory for Neuro Imaging at the University of Southern California.

\section{Conflict of Interest}

The authors have no known or potential conflicts of interest with any people or organizations within 3 years of beginning the submitted work that could inappropriately influence or be perceived to influence their work, including any financial, personal, or other relationships. No conflicts have been identified.

\section{Role of Authors}

All authors had full access to all the data in the study and take responsibility for the integrity of the data and the accuracy of the data analysis. FK programmed the LMEM model and drafted the manuscript, FM performed the statistical analysis, and AS provided neuroanatomical advice and interpretation. 


\section{References}

ADNI General Procedure Manual. http://adni.loni.usc.edu/wp-content/uploads/2008/07/adni2-proceduresmanual.pdf. (accessed: July 21, 2016).

ADNI MRI Scanner Protocols. http://adni.loni.usc.edu/methods/documents/mri-protocols/ (accessed: July 21, 2016).

Amlien I.K., Fjell A.M. (2014) Diffusion Tensor Imaging of White Matter Degeneration in Alzheimer's Disease and Mild Cognitive Impairment.Neuroscience 276, 206-215.

Arnold S.E., Hyman B.T., Flory J., Damasio A.R., van Hoesen G.W. (1991) The topographical and neuroanatomical distribution of neurofibrillary tangles and neuritic plaques in the cerebral cortex of patients with Alzheimer's disease. Cerebral Cortex 1, 103-116.

Ashburner J., Friston K.J. (2000) Voxel-Based Morphometry - The Methods. NeuroImage 11, 805-821.

Basser P.J., Pierpaoli C. (1996) Microstructural and physiological features of tissues elucidated by quantitativediffusion-tensor MRI. Journal of Magnetic Resonance 111, 209-219.

Beckmann C.F., Jenkinson M., Smith S.M. (2003) General multilevel linear modeling for group analysis in FMRI. NeuroImage 20, 1052-1063.

Benjamini Y., Hochberg Y. (1995) Controlling the false discovery rate: a practical approach to multiple testing. Journal of the Royal Statistical Society 57, 289-300.

Bennett I.J., Madden D.J., Vaidya C.J., Howard D.V., Howard J.H. (2010) Age-Related Differences in Multiple Measures of White Matter Integrity: A Diffusion Tensor Imaging Study of Healthy Aging. Human Brain Mapping 31:378-390.

Bernal-Rusiel J.L., Greve D.N., Reuter M., Fischl B., Sabuncu M.R. (2013) Statistical analysis of longitudinal neuroimage data with Linear Mixed Effects models.

Braskie M.N., Toga A.W., Thompson P.M. (2013) Recent Advances in Imaging Alzheimer's Disease. Journal of Alzheimer's Disease 33, S313-S327.

Cho Z.H. (2010) 7.0 Tesla MRI brain atlas. Springer, New York.

Chua T.C., Wen W., Slavin M.H., Sachdev P.S. (2008) Diffusion tensor imaging in mild cognitive impairment and Alzheimer's disease: a review. Current Opinion in Neurology 21, 83-92.

Damoiseaux J.S., Smith S.M., Witter M.P., Sanz-Arigita E.J., Barkhof F., Scheltens P., Stam C.J., Zarei M., Rombouts S.A. (2009) White matter tract integrity in aging and Alzheimer's disease. Human Brain Mapping 30, $1051-1059$.

Douaud G., Jbabdi S., Behrens T.E.J., Menke R.A., Gass A., Monsch A.U., Rao A., Whitcher B., Kindlmann G., Matthews P.M., Smith S. (2011) DTI measures in crossing-fibre areas: Increased diffusion anisotropy reveals early white matter alteration in MCI and mild Alzheimer's disease. NeuroImage 55, 880-890.

Douaud G., Menke R.A.L., Gass A., Monsch A.U., Rao A., Whitcher B., Zamboni G., Matthews P.M., Sollberger M., Smith S. (2013) The Journal of Neuroscience 33, 2147-2155.

Driscoll I., Davatzikos C., An Y., Wu X. Shen D., Kraut M., Resnick S.M. (2009) Longitudinal pattern of regional brain volume change differentiates normal aging from MCI. Neurology 72, 1906-1913.

Fellgiebel A., Yakushev I. (2011) Diffusion tensor imaging of the hippocampus in MCI and early Alzheimer's disease. Journal of Alzheimer's Disease 26, 257-262.

Fillard P., Pennec X., Arsigny V., Ayache N. (2007) Clinical DT-MRI Estimation, Smoothing, and Fiber Tracking With Log-Euclidean Metrics. IEEE Transactions on Medical Imaging 26, 1476-1482.

Fonov V.S., Evans A.C., McKinstry R.C., Almli C.R., Collins D.L. (2009) Unbiased non-linear average ageappropriate brain templates from birth to adulthood. NeuroImage 47, S102.

Friston K.J., Worsley K.J., Frackowiak R.S.J., Mazziotta J.C., Evans A.C. (1993) Assessing the significance of focal activations using their spatial extent. Human Brain Mapping 1, 210-220.

Galecki A., Burzykowsli T. (2013) Linear Mixed-Effects Models Using R. Springer, Heidelberg.Gao Y., Wee C.Y, Kim M., Giannakapoulos P., Montandon M.L., Haller S., Shen D. (2015) MCI Identification by Joint Learning on Multiple MRI Data. Proceedings of the $18^{\text {th }}$ Conference Medical Imaging and Computer-Assisted Intervention, pp. 78-85, Springer, Heidelberg.

Halekoh U., Hojsgaard S. (2014) A Kenward-Roger approximation and parametric boot-strap methods for tests in linear mixed models - the R package pbkrtest. Journal of Statistical Software 59, 1-32.

Hentschel S., Kruggel F. (2004) Determination of the intracranial volume: a registration approach. In: Jiang T. (Ed.): International Workshop on Medical Imaging and Augmented Reality (MIAR 2004), Lecture Notes in Computer Science Vol. 3150, pp. 253-260, Springer, Berlin.

Hinton D.R., Sadun A.A., Blanks J.C., Miller C.A. (1986) Optic-Nerve Degeneration in Alzheimer's Disease. The New England Journal of Medicine 15, 485-487.

Hornik K. (2014) R FAQ. http://CRAN.R-project.org/doc/FAQ/R-FAQ.html (accessed: July 21, 2016). 
Hsu J.L., Leemans A., Bai C.H., Lee C.H., Tsai Y.F. Chiu H.C., Chen W.H. (2008) Gender differences and agerelated white matter changes of the human brain: A diffusion tensor imaging study. NeuroImage 39, 566-577.

Huang H., Fan X., Weiner M., Martin-Cook K., Xiao G., Davis J., Devous M., Rosenberg R., Diaz-Arrastia R. (2012) Distinctive disruption patterns of white matter tracts in Alzheimer's disease with full diffusion tensor characterization. Neurobiology of Aging, 33, 2029-2045.

Hyman B.T., van Hoesen G.W., Kromer L.J., Damasio A.R. (1986) Perforant pathway changes and the memory impairment of Alzheimer's disease. Annals of Neurology 20, 472-481.

Inano S., Takao H., Hayashi N., Abe O., Ohtomo K. (2011) Effects of Age and Gender on White Matter Integrity. American Journal of Neuroradiology 32, 2103-2109.

Ingalhalikar M., Smith A., Parker D., Satterthwaite T.D., Elliott M.A., Ruparel, K., Harkonarson H., Gur R.E., Gur R.C., Verma R. (2014) Sex differences in the structural connectome of the human brain. Proceedings of the National Academy of Sciences of the United States of America 111, 823-828.

Johnson N.L., Rogers C.A. (1951) The moment problem for unimodal distributions. Annals of Mathematical Statistics, 22, 433-439.

Kantarci K., Petersen R.C., Boeve B.F., Knopman D.S., Weigand S.D., O'Brien P.C., Shiung M.M., Smith G.E., Ivnik R.J., Tangalos E.G., Jack C.R. (2005) DWI predicts future progression to Alzheimer disease in amnestic mild cognitive impairment. Neurology 64, 902-904.

Kanaan R.A., Allin M., Picchioni M., Barker G.J., Daly E., Shergill S.S., McGuire P.K. (2012) Gender Differences in White Matter Microstructure. PLoS One 7, e38272.

Kanaan R.A., Chaddock C., Allin M., Picchioni M.M., Daly E., Shergill S.S., McGuire P.K. (2014) Gender Influence on White Matter Microstructure: A Tract-Based Spatial Statistics Analysis. PLoS One 9, e91109.

Katanoda K., Matsuda Y., Sugishita M. (2002) A Spatio-temporal Regression Model for the Analysis of Functional MRI Data. NeuroImage 17, 1415-1428.

Keihaninejad S., Zhang H., Ryan N.S., Malone I.B., Modat M., Cardoso H.J., Cash D.M., Fox N.C., Ourselin S. (2013) An unbiased longitudinal analysis framework for tracking white matter changes using diffusion tensor imaging with application to Alzheimer's disease. NeuroImage 72, 153-163.

Kenward M.G., Roger J.H. (1997) Small Sample Inference for Fixed Effects from Restricted Maximum Likelihood. Biometrics 53, 983-997.

Kitamura S., Kiuchi K., Taoka T., Hashimoto K., Ueda S., Yasuno F., Morikawa M., Kichikawa K., Kishimoto T. (2013) Longitudinal white matter changes in Alzheimer's disease: A tractography-based analysis study. Brain Research $1515,12-18$.

Kohannim O., Hua X., Hibar D.P., Lee S., Chou Y.Y., Toga A.W., Jack C.R., Weiner M.W., Thompson P.M. (2010) The Alzheimer's Disease Neuroimaging Initiative, Boosting power for clinical trials using classifiers based on multiple biomarkers. Neurobiology of Aging 31, 1429-1442

Kovalev V.A., Kruggel F., von Cramon D.Y. (2003) Gender and Age Effects in Structural Brain Asymmetry as Measured by MRI Texture Analysis. NeuroImage 19, 895-905.

Kruggel F., von Cramon D.Y. (1999) Alignment of magnetic-resonance brain data sets with the stereotactic coordinate system. Medical Image Analysis 3, 1-11.

Kruggel F., D.Y. von Cramon (1999) Modeling the hemodynamic response in single-trial functional MRI experiments. Magnetic Resonance in Medicine 42, 787-797.

Kruggel F., Turner J., Muftuler L.T. (2010) Impact of scanner hardware and imaging proto-col on image quality and compartment volume precision in the ADNI cohort. NeuroImage 49, 2123-2133.

Kruggel F. (2016) The BRIAN system. http://sip.eng.uci.edu/index.php?page=brian (accessed: July 21, 2016).

Laird N., Lange N., Stram D. (1987) Maximum Likelihood Computations with Repeated Measures: Application of the EM Algorithm. Journal of the American Statistical Association 82, 97-105.

Lin M., He H., Schiffito G., Zhong J. (2016) Simulation of changes in diffusion-related to different pathologies at cellular level after traumatic brain injury. Magnetic Resonance in Medicine 76, 290-300.

Lindstrom M.J., Bates D.M. (1988) Newton-Raphson and EM Algorithms for Linear Mixed-Effects Models for Repeated-Measures Data. Journal of the American Statistical Association 83, 1014-1022.

Liu F., Suk H.I., Wee C.Y., Chen H., Shen D. (2013) High-Order Graph Matching Based Feature Selection for Alzheimer's Disease Identification. Proceedings of the $16^{\text {th }}$ Conference Medical Imaging and ComputerAssisted Intervention, pp. 319-328, Springer, Heidelberg.

Madden D.J., Bennett I.J., Burzynska A., Potter G.G., Chen N.K., Song A.W. (2012) Diffusion tensor imaging of cerebral white matter integrity in cognitive aging. Biochimica et Biophysica Acta 1822, 386-400.

Maes F., Maes A., Collignon D., Vandermeulen D., Marchal A., Suetens P. (1997). Multi-modality image registration by maximization of mutual information. IEEE Transactions on Medical Imaging 16, 187-198.

Mapstone M., Cheema A.K., Fiandaca M.S., Zhong X., Mhyre T.R., MacArthur L.H., Hall W.J., Fisher S.G., 
Peterson D.R., Haley J.M., Nazar M.D., Rich S.A., Berlau D.J., Peltz C.B., Tan M.T., Kawas C.H., Federoff H.J. (2014) Plasma phospholipids identify antecedent memory impairment in older adults. Nature Medicine 20, 415-418.

McEvoy L.K., Fennema-Notestine C., Roddey J.C., Hagler D.J., Holland D., Karow D.S., Pung C.J., Brewer J.B., Dale A.M. (2009) Alzheimer Disease: Quantitative Structural Neuroimaging for Detection and Prediction of Clinical and Structural Changes in Mild Cognitive Impairment. Radiology 251, 195-205.

McKhann G.M., Knopman D.S., Chertkow H., Hyman B.T., Jack C.R., Kawas C.H., Klunk W.E., Koroshetz W.J., Manly J.J., Mayeux R., Mohs R.C., Morris J.C., Rossor M.N., Scheltens P., Carrillo M.C., Thies B., Weintraub S., Phelps C.H. (2011) The diagnosis of dementia due to Alzheimer's disease: Recommendations from the National Institute on Aging-Alzheimer's Association workgroups on diagnostic guidelines for Alzheimer's disease. Alzheimer's \& Dementia 7. 263-269.

Morris J.C., Roe C.M., Grant E.A., Head D., Storandt M., Goate A.M., Fagan A.M., Holtzman D.M., Mintun M.A. (2009) Pittsburgh compound B imaging and prediction of progression from cognitive normality to symptomatic Alzheimer disease. Archives of Neurology 66, 1469-1475.

Molinuevo J.L., Ripolles P., Simo M., Llado A., Olives J., Balasa M., Antonell A., Rodriguez-Fornells A., Rami L. (2014) White matter changes in preclinical Alzheimer's disease: a magnetic resonance imaging-diffusion tensor imaging study on cognitively normal older people with positive amyloid b protein 42 levels. Neurobiology of Aging 35, 2671-2680.

Nir T.M., Villalon-Reina J.E., Prasad G., Jahanshad N., Joshi S.H., Toga A.W., Bernstein M.A., Jack Jr C.R., Weiner M.W., Thompson P.M. (2015) Diffusion weighted imaging-based maximum density path analysis and classification of Alzheimer's disease. Neurobiology of Aging 36, 5132-5140.

O’Dwyer L., Lamberton F., Bokde A.L.W., Ewers M., Faluyi Y.O., Tanner C., Mazoyer B., O’Neill D., Bartley M., Collins D.R., Coughlan T., Prvulovic D., Hampel H. (2011) Using Diffusion Tensor Imaging and Mixed-Effects Models to Investigate Primary and Secondary White Matter Degeneration in Alzheimer's Disease and Mild Cognitive Impairment. Journal of Alzheimer's Disease 26, 667-682.

Pfefferbaum A., Adalsteinsson E., Rohlfing T., Sullivan E.V. (2010) Diffusion tensor imaging of deep gray matter brain structures: Effects of age and iron concentration. Neurobiology of Aging 31. 482-493.

Ravetti M.G., Moscato P. (2008) Identification of a 5-Protein Biomarker Molecular Signature for Predicting Alzheimer's Disease. PLoS One 10, e1371.

Resnick S.M., Pham D.L., Kraut M.A., Zonderman A.B., Davatzikos C. (2003) Longitudinal Magnetic Resonance Imaging Studies of Older Adults: A Shrinking Brain. The Journal of Neuroscience 23, 3295-3301.

Rogalski E.J., Murphy C.M., deToledo-Morrell L., Shah R.C., Moseley M.E., Bammer R., Stebbins G.T. (2009) Changes in parahippocampal white matter integrity in amnestic mild cognitive impairment: A diffusion tensor imaging study. Behavioural Neurology 21, 51-61.

Sadun A., Brassi C.J. (1990) Optic Nerve Damage in Alzheimer's Disease. Ophthalmology 97, 9-17.

Salat D.H., Tuch D.S., van der Kouwe A.J.W., Greve D.N., Pappu V., Lee S.Y., Hevelone N.D., Zaleta A.K., Growdon J.H., Corkin S., Fischl B., Rosas H.D. (2010) White matter pathology isolates the hippocampal formation in Alzheimer's disease. Neurobiology of Aging 31, 244-256.

Scheltens P., Barkhof D., Leys D., Wolters E.C., Ravid R., Kamphorst W. (1995) Histopathologic correlates of white matter changes on MRI in Alzheimers' disease and normal aging. Neurology 45 883-888.

Schumann C.M., Bloss C.S., Barnes C.C., Wideman G.M., Carper R.A., Akshoomoff A., Pierce K., Hagler D., Schork N., Lord C., Courchesne E. (2010) Longitudinal Magnetic Resonance Imaging Study of Cortical Development through Early Childhood in Autism The Journal of Neuroscience 30, 4419-4427.

Sexton C.E., Walhovd K.B., Storsve A.B., Tamnes C.K., Westlye L.T., Johansen-Berg H., Fjell A.M. (2014) Accelerated Changes in White Matter Microstructure during Aging: A Longitudinal Diffusion Tensor Imaging Study. The Journal of Neuroscience 34, 15425- 15436.

Solodkin A., Chen E.E., van Hoesen G.W., Heimer L., Shereen A., Kruggel F., Mastrianni J. (2013) In vivo parahippocampal white matter pathology as a biomarker of disease progression to Alzheimer's disease. Journal of Comparative Neurology 18, 4300-4317.

Suk H.I., Shen D. (2014) Clustering-Induced Multi-task Learning for AD/MCI Classification.Proceedings of the $17^{\text {th }}$ Conference Medical Imaging and Computer-Assisted Intervention, pp. 393-400, Springer, Heidelberg.

Sullivan E.V., Pfefferbaum A. (2006) Methodological and Conceptual Advances in the Study of Brain-Behavior Dynamics: A Multivariate Lifespan Perspective. Neuroscience \& Biobehavioral Reviews 30, 749-761.

Sullivan E.V., Pfefferbaum A. (2007) Neuroradiological characterization of normal adult ageing. British Journal of Radiology 80, S99-S108.

Teipel S.J., Stahl R., Dietrich O., Schoenberg S.O., Perneczky R., Bokde A.L., Reiser M.F., Moller H.J., Hampel H. (2007) Multivariate network analysis of fiber tract integrity in Alzheimer's disease. NeuroImage 34, 985-995. 
Teipel S.J., Meindl T., Wagner M., Stieltjes B., Reuter S., Hauenstein K.H., Filippi M., Ernemann U., Reiser M.F., Hampel H. (2010) Longitudinal changes in fiber tract integrity in healthy aging and mild cognitive impairment: a DTI follow-up study. Journal of Alzheimer's Disease 22, 507-522.

Teipel S.J., Grothe M.J., Filippi M., Fellgiebel A., Dyrba M., Frisoni G.B., Meindl T., Bokde A.L.W., Hampel H., Klöppel S., Hauenstein K. (2014) Fractional Anisotropy Changes in Alzheimer's Disease Depend on the Underlying Fiber Tract Architecture: A Multiparametric DTI Study using Joint Independent Component Analysis. Journal of Alzheimer's Disease, doi: 10.3233/JAD-131829.

Vercauteren T., Pennec X., Perchant A., Ayache N. (2009) Diffeomorphic demons: Efficient non-parametric image registration. NeuroImage 49, S61-S72.

Villain N., Fouquet M., Baron J.C., Mezenge F., Landeau B., de La Sayette V., Viader F., Eustache F., Desgranges B., Chetelat G. (2010) Sequential relationships between grey matter and white matter atrophy and brain metabolic abnormalities in early Alzheimer's disease. Brain 133, 3301-3314.

Vlassenko A.G., McCue L., Jasielec M.S., Su Y., Gordon B.A., Xiong C., Holtzman D.M., Benzinger T.L., Morris J.C., Fagan A.M. (2016) Imaging and cerebrospinal fluid biomarkers in early preclinical Alzheimer disease. Ann Neurol. 2016 Jul 11. doi: 10.1002/ana.24719.

Wang Q., Xu X., Zhang M. (2010) Normal aging in the basal ganglia evaluated by eigenvalues of Diffusion Tensor Imaging. American Journal of Neuroradiology 31, 516-520.

Wang T., Shi F., Jin Y., Yap P.T., Wee C.Y., Zhang J., Yang C., Li X., Xiao S., Shen D. (2016) MultilevelDeficiency of White Matter Connectivity Networks in Alzheimer's Disease: A Diffusion MRI Study with DTI and HARDI Models. Neural Plasticity, doi: 10.1155/2016/2947136.

Weiner M.W., Veitch D.P., Aisen P.S., Beckett L.A., Cairns N.J., Green R.C., Harvey D., Jack C.R., Jagust W., Liu E., Morris J.C., Petersen R.C., Saykin A.J., Schmidt M.E., Shaw L., Siuciak J.A., Soares H., Toga A.W., Trojanowski J.Q. (2012) The Alzheimer's Disease Neuroimaging Initiative: A review of papers published since its inception. Alzheimer's \& Dementia 8, S1-S68.

Yassa M.A., Muftuler L.T., Stark C.E. (2010) Ultrahigh-resolution microstructural diffusion tensor imaging reveals perforant path degradation in aged humans in vivo. Proceedings of the National Academy of Sciences of the United States of America 107, 12687- 12691.

Zhan L., Zhou J., Wang Y., Jin Y., Jahanshad N., Prasad G., Nir T.M., Leonardo C.D., Ye J., Thompson P.M. (2015) Comparison of nine tractography algorithms for detecting abnormal structural brain networks in Alzheimer's disease. Frontiers in Aging Neuroscience, doi: 10.3389/fnagi.2015.00048

Zu C., Jie B., Liu M., Chen S., Shen D. (2015) Label-aligned multi-task feature learning for multimodal classification of Alzheimer's disease and mild cognitive impairment. Brain Imaging and Behavior doi: 10.1007/s11682-015-9480-7. 


\section{Figures}

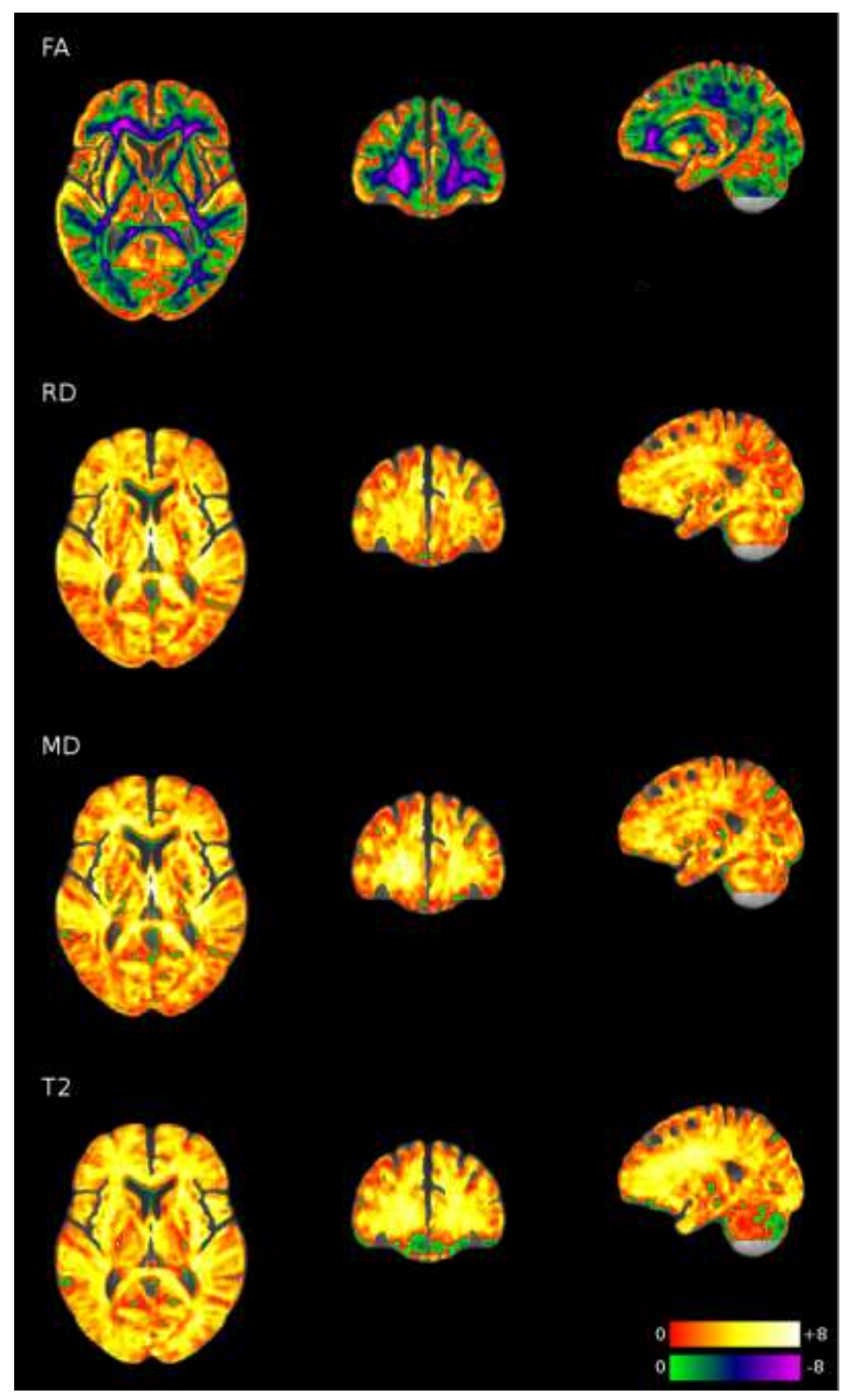

Figure 1: Influence of aging on WM, as shown by un-thresholded $z$-score maps determined from fractional anisotropy (FA), radial diffusivity (RD), mean diffusivity (MD), and $\mathrm{T}_{2}$ intensity (T2). Positive $\mathrm{z}$-scores range from 0 (red) to 8 (white), negative $\mathrm{z}$-scores from 0 (green) to -8 (magenta). A general pattern of age-related FA decrease, RD, MD and T2 increase is found in most WM compartments. Besides, a second pattern of FA increase and a moderate RD, MD and T2 increase is found in cortical GM and basal ganglia as well as some WM areas. For detail information from selected voxels, refer to Table 1. 


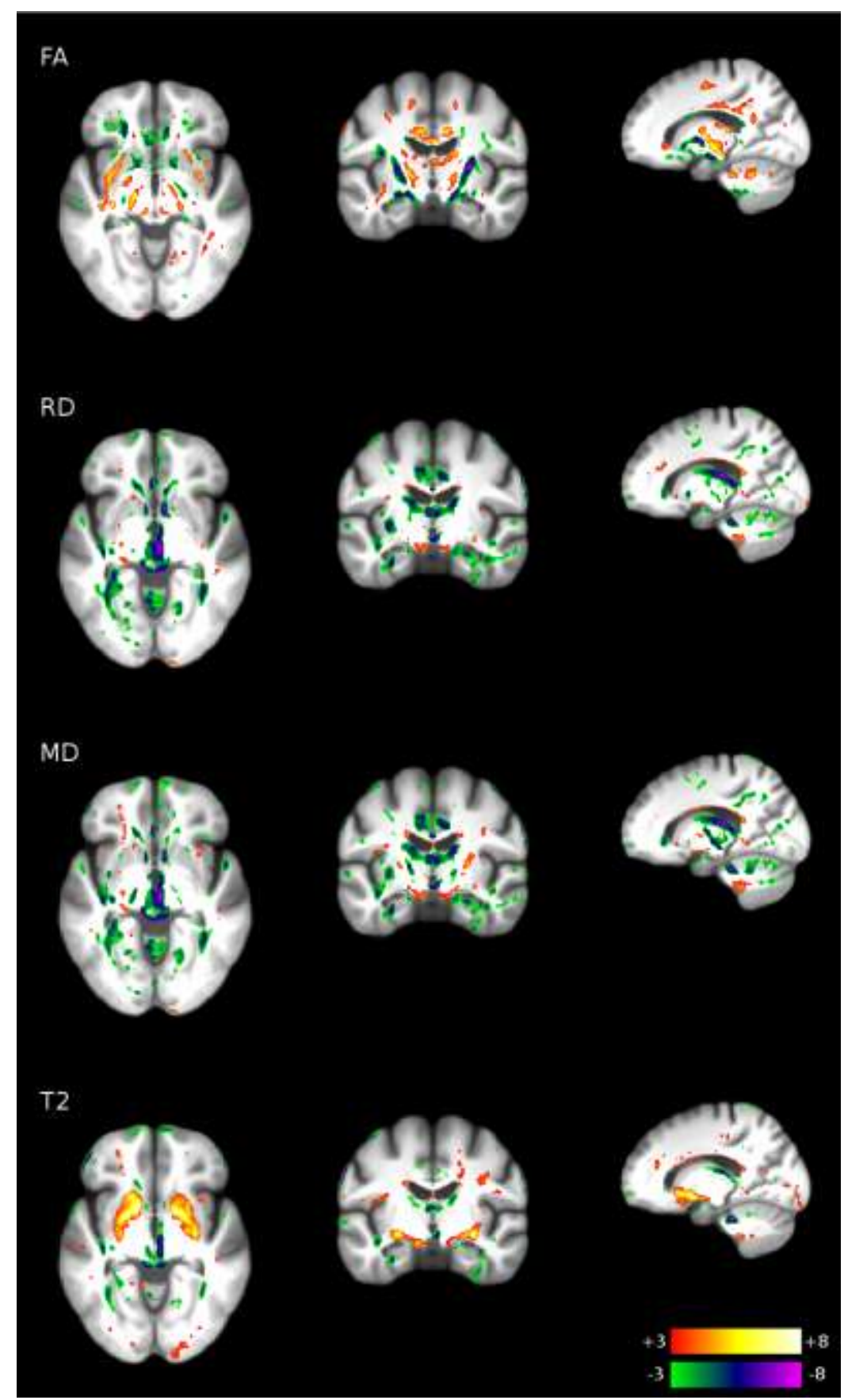

Figure 2: Differences between males and females, as determined from fractional anisotropy (FA), radial diffusivity (RD), mean diffusivity (MD), and $\mathrm{T}_{2}$ intensity (T2). Shown are thresholded $\mathrm{z}-$ score maps, with positive z-scores ranging from 3 (red) to 8 (white), negative z-scores from -3 (green) to -8 (magenta). Regions shown are corrected for multiple comparison by controlling the family-wise error rate. For detail information of these regions, refer to Table 2. 


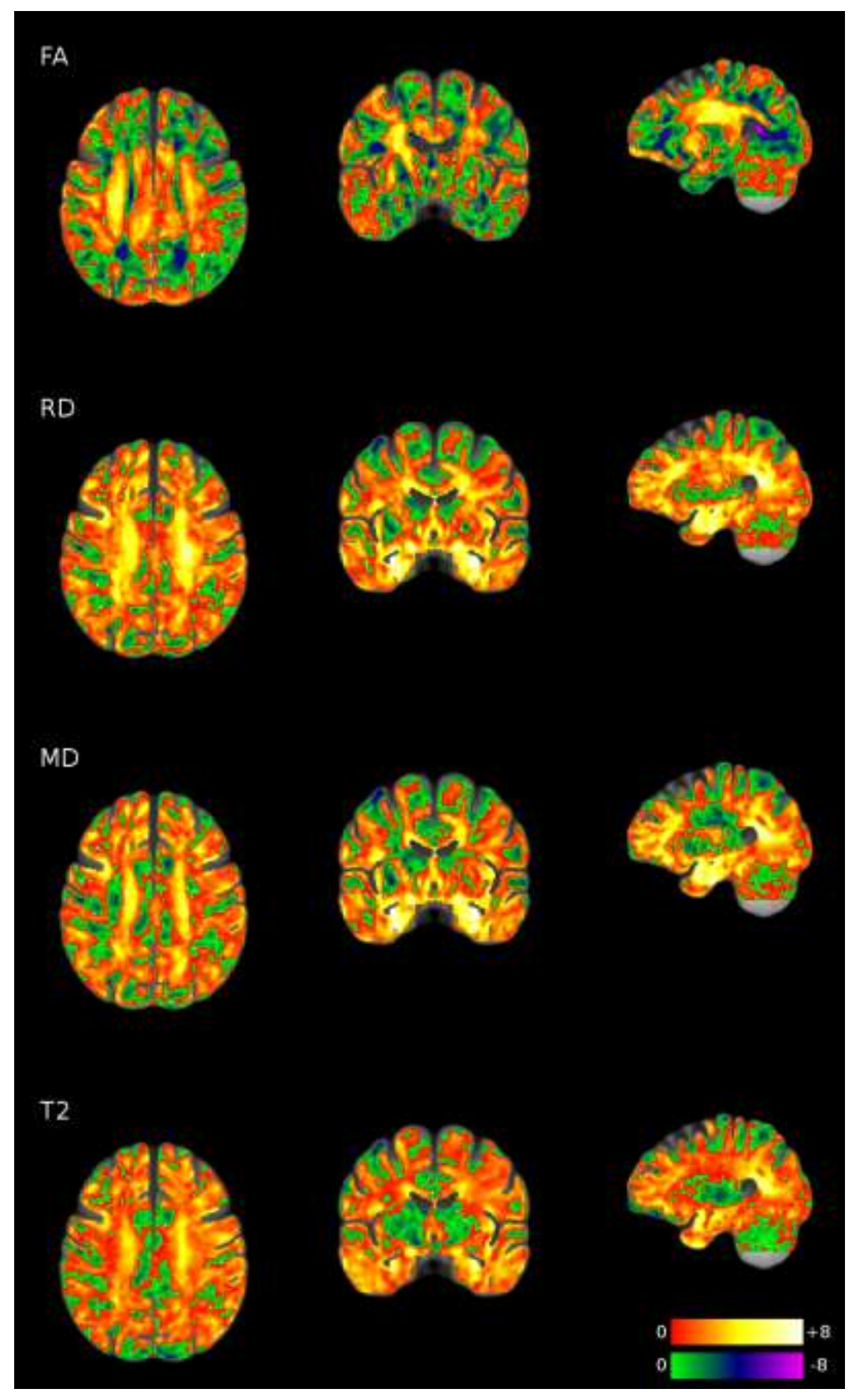

Figure 3: Difference between AD patients and healthy controls, as determined from fractional anisotropy (FA), radial diffusivity (RD), mean diffusivity (MD), and $\mathrm{T}_{2}$ intensity (T2). Shown are un-thresholded $\mathrm{z}$-score maps, with positive $\mathrm{z}$-scores ranging from 0 (red) to 8 (white), negative z-scores from 0 (green) to -8 (magenta). For detail information from selected voxels, refer to Table 3. 

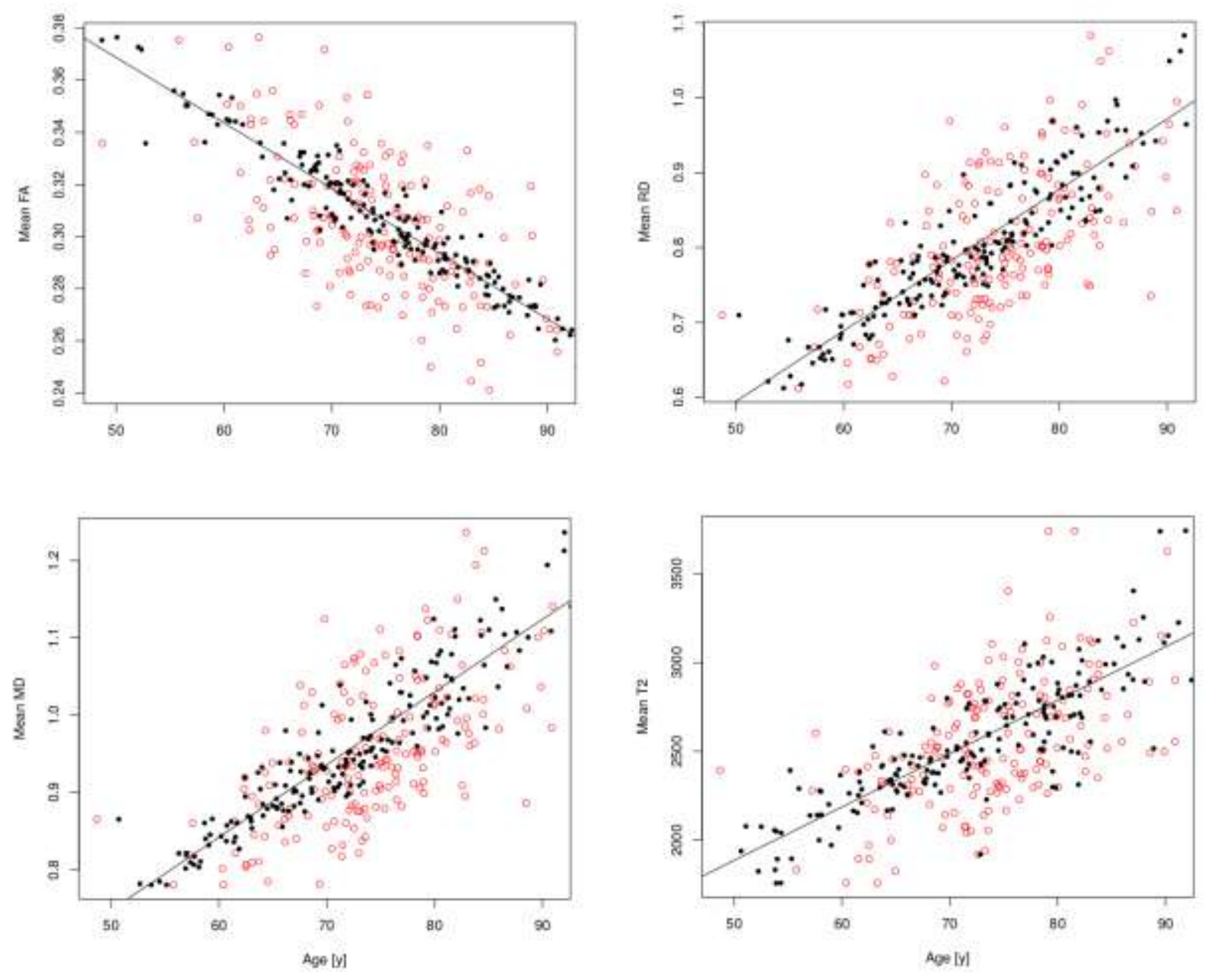

Figure 4: Age-related changes in FA (top left), RD (top right), MD (below left) and T2 (below right). Red circles corresponds to subject-wise means, black dots to subject-wise means adjusted for the proposed individual difference in the aging process. Note that adjusted data are well modeled by a linear age relationship. 


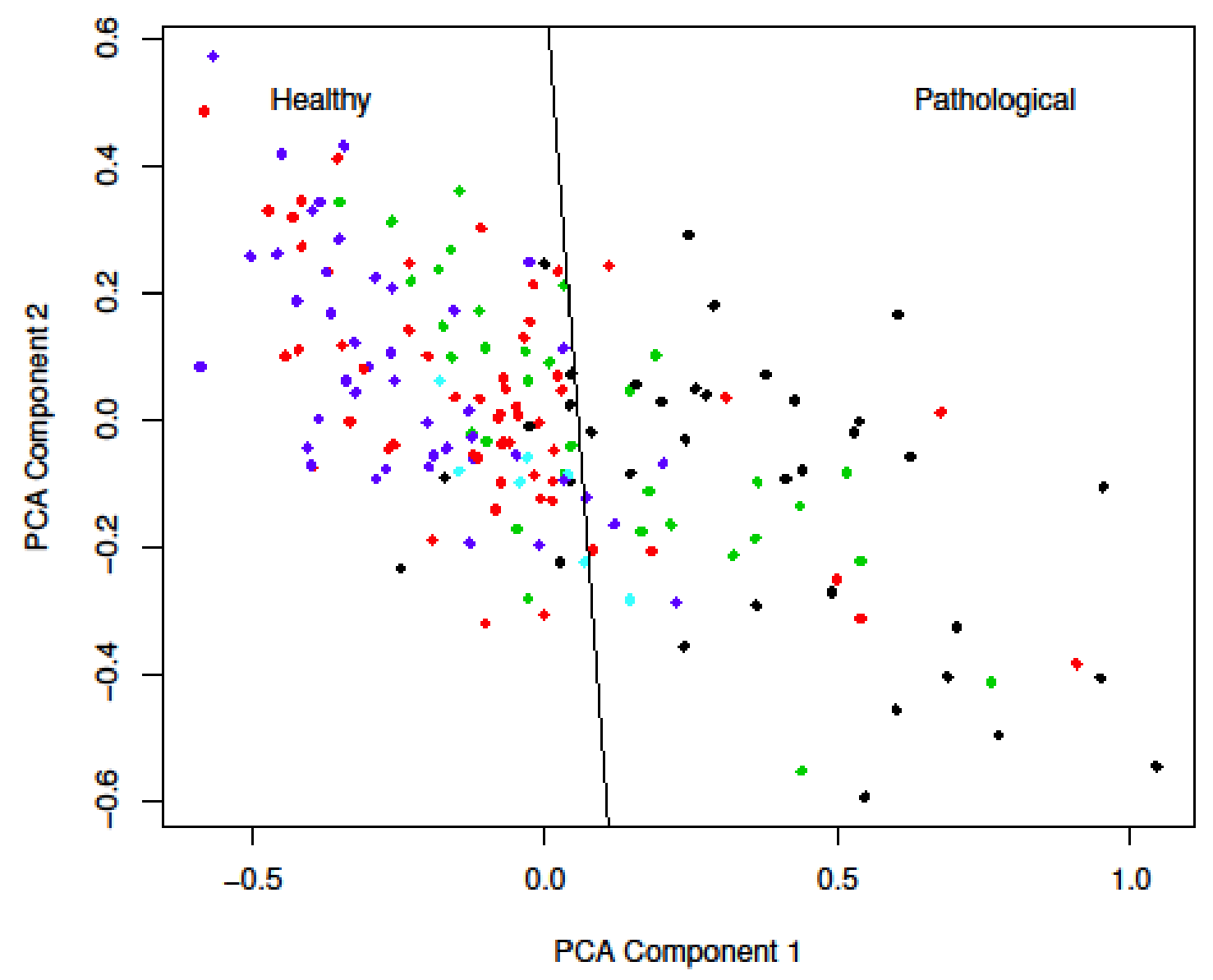

Figure 5: Evaluation of individual "health scores" in a region-of-interest in the anterior mesial temporal lobe. Each dot corresponds to a subject average, color-coded by clinical group (AD: black; EMCI: red; LMCI: green; CN: blue; SMC: light blue). A simple linear classifier based on PCA-projected data yields a very high predictive power of 0.991 for distinguishing AD patients from healthy controls. Note that some MCI patients are clearly mapped into the pathological domain. 


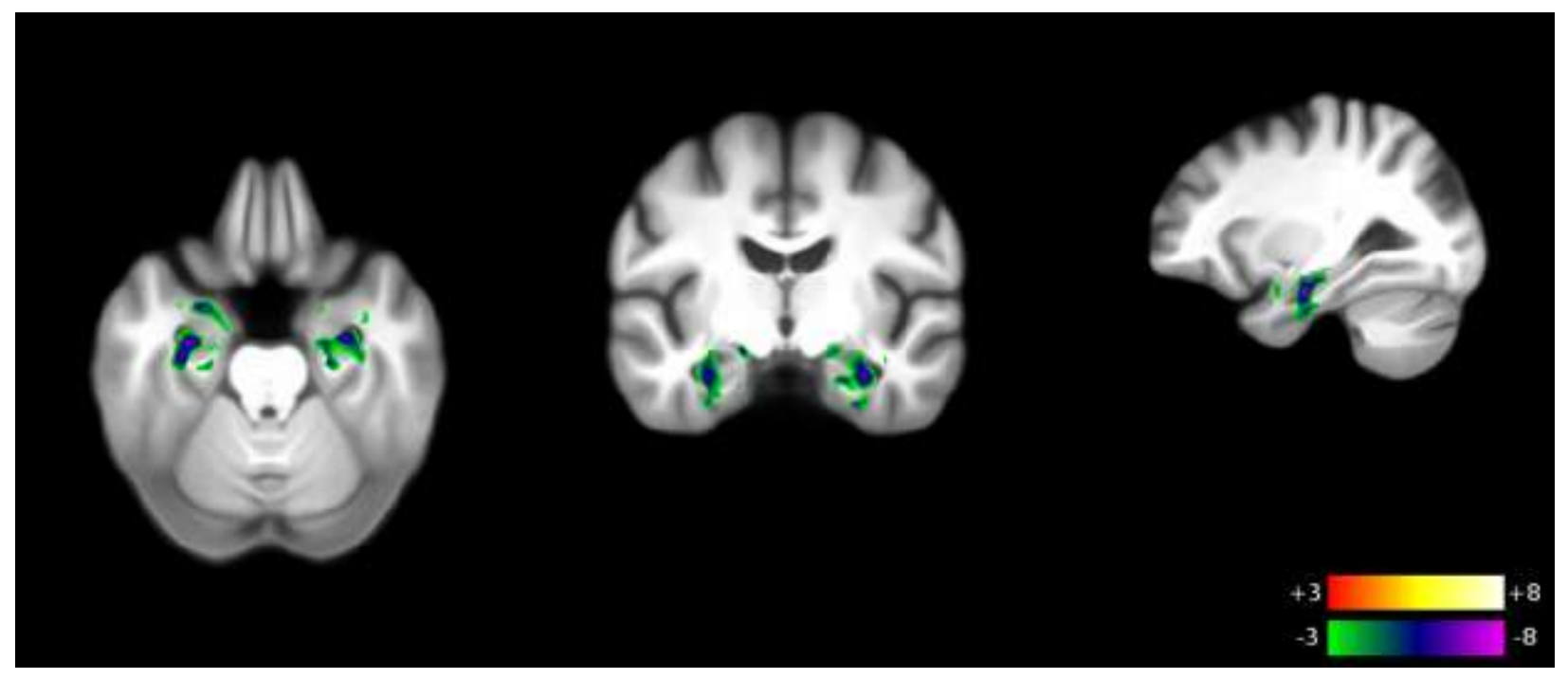

Figure 6: Voxels that discriminate best between healthy controls and AD patients were found in over-projection with white matter fiber structures of the hippocampus and parahippocampus, e.g., the alveus and perforant path. Interestingly, the optic tract on both sides was also found as discriminatory. 


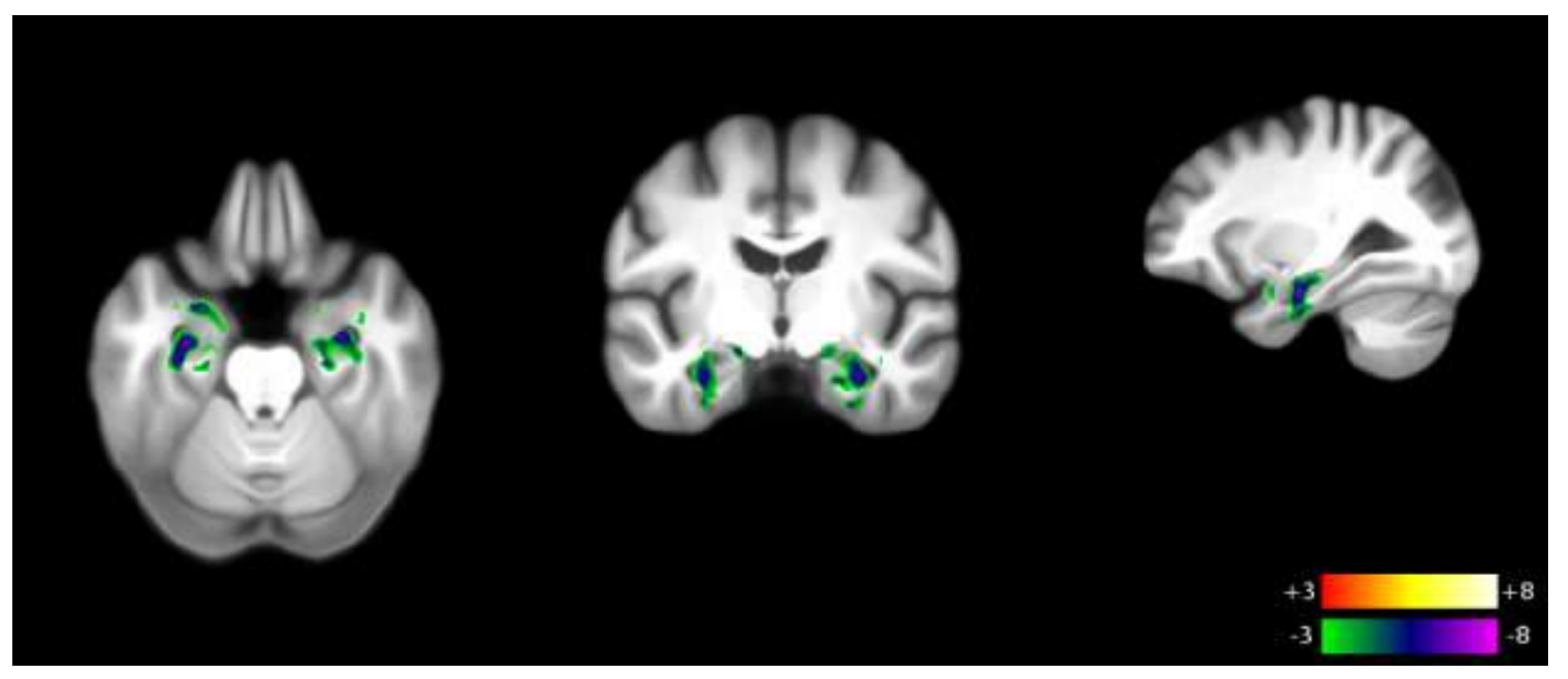

Diffusion-weighted MR imaging provides promising parameters that are suggestive of a biomarker for the early detection of Alzheimer's Disease. Highly discriminative white matter fiber structures belong to the hippocampus and parahippocampus, e.g., the alveus and perforant path. 